\title{
MITTEILUNGEN
}

\section{Zum 60. Geburtstag von Hermann Holthusen}

$\mathrm{A}^{\mathrm{n}}$ 22. September 1946 jährte sich zum 60. Male der Geburtstag von Hermann Holthusen, dem die Strahlenheilkunde in Deutschland im wesentlichen die Richtung verdankt, die sie in den letzten drei Jahrzehnten genommen hat. Nach beendetem Medizinstudium kam er bereits als Volontärassistent von Albérs-Schönberg mit der Röntgenologie in Berührung, die für ihn Weg und Ziel seiner Lebensarbeit werden sollte. Während er als Assistent von Ludwig Krehl ein Röntgeninstitut in Heidelberg einrichtete, arbeitete er zugleich am Len ardschen Institut, gemeinsam mit Ramsauer und Becker, über das Problem der Radium-Emanation.

Mit der raschen technischen Entwicklung leistungsfähiger Röntgenstrahlenquellen in den Jahren vor und während des ersten Weltkrieges hielt der Anstieg der Heilerfolge nicht Schritt, da es an einer geeigneten Dosierungsmethode fehlte. Zwar war dieses Problem schon frühzeitig erkannt und viel bearbeitet worden, wobei $\mathrm{Holzknecht,} \mathrm{Sa-}$ bouraud-Noiret, Kienböck chemische Methoden, Christen, Friedrich, Dessauer, Solom on Ionisationsmethoden, Fürsten a u die Leitfähigkeitsänderung von Selen und Seitz und Wintz sowie Jüngling biologische Methoden verwendeten. Trotzdem gelang es nicht, eine Methode zu entwickeln, die zu einer allgemein anerkannten Einheit hätte führen können. So kam es, daß nach Beendigung des Weltkrieges noch immer recht erhebliche Unterschiede in den Ergebnissen der verschiedenen Meßmethoden auftraten. In diese Zeit fällt Holthusens Arbeit ,Über die Bedingungen der Röntgenstrahlenenergiemessungen bei verschiedenen Impulsbreiten auf luftelektrischem Wege“1. Mit bewundernswerter Klarheit wird dort schon ausgesprochen, daß nur eine einwandfreie, jederzeit und an jedem Orte nach einer bestimmten Vorschrift reproduzierbare Einheit als Grundlage für die Röntgenstrahlen-Dosierung gelten kann und hierfür nur die Ionisationsmethode in Betracht kommt. Durch die Entwicklung der „Faßkammer“ als Meßgerät schuf $\mathrm{H}$ olth u se n gleichzeitig das Standardinstrument, das dieser Me-

1 Fortschr. Gebiete Röntgenstrahlen 16, 211 [1919]. thode die zum Ziel führende Richtung gab. Auf diesem Wege fortschreitend, gelangten $\mathrm{K} \ddot{\mathrm{u}} \mathrm{s}$ tner, Berg, Schwerdtfeger und Thaller, Behnken, Solomon, Taylor u. a. zu ihren bekannten Ergebnissen, die schließlich in der Schaffung der physikalisch exakt definierten Dosiseinheit „Röntgen“ durch Behnken ihren Abschluß fanden.

Wenn die neugeschaffene Einheit, zunächst nach den Vorschlägen der Physikalisch-Technischen Reichsanstalt in Deutschland eingeführt, raschen Eingang in die Strahlenheilkunde fand, so ist dies zum großen Teil Holthusen zu verdanken, dessen Autorität gleicherweise von den Röntgenphysikern wie auch den praktisch tätigen Röntgenologen vorbehaltlos anerkannt wurde. Auch der Internationale Radiologenkongreß entschloß sich 1928 in Stockholm für die Einheit „Röntgen“, wobei es hier ohne Bedeutung ist, daß die von Behnken aufgestellte Einheit durch Rückführung der Bezugstemperatur auf $0^{\circ} \mathrm{C}$ eine Korrektur um rund $6 \%$ erfuhr.

Mit der Schaffung der physikalischen Röntgeneinheit war für die Bestimmung einer medizinisch verabreichten Strahlenmenge ein erster und notwendiger Schritt getan worden; er genügte jedoch nicht, um die Bedürfnisse der Strahlenheilkunde hinsichtlich des Dosierungsproblems voll zu befriedigen, worauf $\mathrm{Holth}$ u sen als einer der ersten hinwies. Durch neue experimentelle Arbeiten zeigte er den Weg, um von der ,,physikalischen“ zur ,,biologischen“" Dosiseinheit zu gelangen. Dabei baute Holthusen Methoden zur quantitativen Bestimmung biologischer Strahlenreaktionen aus, wobei er insbesondere die von Perthes in die Strahlenbiologie eingeführten Askariseier verwendete. Die Ergebnisse seiner Arbeiten über die Wellenlängenabhängigkeit der biologischen Reaktion, den Zeitfaktor u. a. stellen einen wesentlichen Bestandteil der experimentellen Grundlagen dar, auf denen die heutigen treffertheoretischen Anschauungen über das Zustandekommen einer biologischen Strahlenwirkung beruhen. $\mathrm{Zu}$ gleicher Zeit bearbeitete Holthusen eine große Reihe strahlenphysiologischer Probleme, die, wie u. a. die neuesten Ar- 
beiten schweizerischer Forscher (Liechti, Wilbrandt) lehren, auch heute noch von Bedeutung sind. Für Holthusen als Arzt stand schon frühzeitig das Krebsproblem im Mittelpunkt des Interesses. Was er auf diesem Gebiete zusammen mit bewährten Mitarbeitern unter erschwerten äußeren Umständen geleistet hat, bezeugen neben zahlreichen Originalarbeiten vor allem auch die $\mathrm{zu}^{-}$ sammenfassenden Berichte und Übersichten, die er auf vielen Kongressen gegeben hat. In diesem $\mathrm{Zu}^{-}$ sammenhang sind auch die gemeinsam mit $\mathrm{Ja}$ cobi und Liechti ausgearbeiteten Tafeln über die Röntgenstrahlengemische zu nennen, durch die der praktisch tätige Röntgenologe auf die große Bedeutung des Begriffes der Halbwertschicht und der Homogenität hingewiesen wird.

In einem gemeinsam mit Braun verfaßten Werke über die „Dosimetrie der Röntgenstrahlen“ hat $\mathrm{Holthusen}$ in neuerer Zeit seine vielfältigen Ergebnisse und reichen Erfahrungen niedergelegt. Damit wurde ein Standardwerk für die Radiologie geschaffen, das in gleicher Weise die physikalischtechnischen als auch die biologisch-medizinischen Fragen behandelt.

Nach dem Vorangegangenen überrascht es nicht, daß der Strahlentherapeut Holthusen bei Verwendung der Radiumstrahlen das Fehlen einer dem „Röntgen“ vergleichbaren Dosiseinheit stets als eine starke Hemmung für den weiteren Ausbau der Radiumtherapie empfand. Er wandte sich daher auch dieser Frage zu mit dem Ziel, die für die Röntgenstrahlen geschaffene Dosiseinheit auch auf die Radiumstrahlen zu übertragen. Da ihm die definitionsgemäße Anwendung der Ionisierungsmethode für die ärztliche Praxis mit den vorhandenen Mitteln nicht als einwandfrei durchführbar erschien, arbeitete er mit seiner Mitarbeiterin $\mathrm{Ha}$ mann ein einfaches photographisches Verfahren aus, das bei einem Vergleich mit dem von Friedrich, Schulze und Henschke entwickelten Ionisationsverfahren nicht nur gute Übereinstimmung ergab, sondern sich bei sehr kleinen Abstän- den diesem Verfahren sogar als überlegen erwies.

So hat sich Holthusen durch sein bisheriges Wirken einzigartige Verdienste um die medizinische Strahlenkunde erworben. Seine unbestechliche, vornehme und zugleich verbindliche Natur, die ihn bei aller Zurückhaltung nicht davon abhält, bei öffentlichen Diskussionen in scharf pointierter, klarer Form Irrtümer richtigzustellen und Wesentliches herauszuarbeiten, hat ihm die besondere Achtung der deutschen und ausländischen Röntgenologen eingetragen. Als großer Vorzug fällt dabei stets ins Gewicht, daß Holthusen in seltener Weise die Sprache des Physikers ebenso beherrscht wie die des Mediziners.

Das Bild von Hermann Holthusen wäre unvollständig, würde man hier nicht noch seiner allgemein menschlichen Neigungen gedenken. Denn der Kreis seines Wirkens und seiner Interessen ist nicht nur auf sein engeres Fachgebiet beschränkt. In den wenigen stillen Stunden, die ihm als Arzt verbleiben, wandert er in die Natur hinaus, um immer aufs neue ihre Offenbarungen zu erleben. Der Harmonie seines Wesens wird durch seine musischen Neigungen Genüge getan, vor allem durch die Musik, die in seinem Hause die Brücke zur Kunst schlägt.

Nachdem der Krieg sein Privatinstitut vernichtet und das von ihm aufgebaute Allgemeine Strahleninstitut St. Georg in Hamburg stark beschädigt hatte, erwuchs Holthusen durch die Leitung eines Ausweichbetriebes der Stadt Hamburg, der Spezial-Geschwulst-Klinik in Bevensen, erneut eine große ärztliche Aufgabe. Daß es ihm trotzdem möglich sein werde, sich auch den vielerlei harrenden Problemen der experimentellen Strahlenbiologie und -physik mit dem großen Schatz seiner langjährigen Erfahrungen weiter zu widmen, ist der herzliche Wunsch all seiner vielen Freunde, Kollegen und Schüler.

Robert $J$ a eger, Hanns Langend orff, Herbert Pychlau. 